\title{
Shaping Requirements for Institutional Web Applications: Experience from an Industrial Project
}

\author{
Vito Perrone $^{1}$, Davide Bolchini ${ }^{2}$, Andrea Rastellini ${ }^{3}$, Luigi Dragone ${ }^{3}$ \\ ${ }^{1}$ HOC laboratories - Politecnico di Milano (Italy) \\ perrone@elet.polimi.it \\ ${ }^{2}$ TEC-Lab - University of Lugano \\ davide.bolchini@lu.unisi.ch \\ ${ }^{3}$ CM Sistemi S.p.A., Roma (Italy) \\ \{andrea.rastellini, luigi.dragone\}@gruppocm.it
}

\begin{abstract}
In this paper we recount the experience and the lessons we learned trying to use concretely and in-depth a requirements engineering method (called AWARE) combined with a conceptual user-centered design method (called W2000) for the development of the Institutional website of the Superior Council for Public Works of the Ministry of Infrastructure and Transportation in Italy. The project is framed within its contractual context, and discussed through the process followed and the artifacts produced during requirements analysis and design. Lessons learned are depicted from a twofold perspective. From the one side they crystallize insights from the research experience of using and transferring the methods to industry, suggesting practical and methodological recommendations. From the other they express what a medium-size software company has experienced trying to use academic methods.
\end{abstract}

Keywords: web applications, goal-oriented requirements analysis, user-centered conceptual design, technology transfer.

\section{Introduction and Project Background}

Despite requirements and design methodologies are increasingly available in the research arena, academia still needs to be further exposed to best practices and real-world project experiences, where existing conceptual tools are tentatively but actually exploited, and where useful recommendations can emerge.

This paper recounts the experience and the lessons we learned applying goal-oriented requirements engineering (in the form of the AWARE model [6]) and user-centered conceptual design (in the form of W2000 method [7] [10]) for the development of an institutional web application for the Italian Ministry of Infrastructure and Transportation.
In Summer 2003, HOC laboratory (Politecnico di Milano) and CM Sistemi ${ }^{1}$ (CM hereafter) met each other in a seminar organized with the purpose of bringing to the attention of the local (Italian) ICT industry the requirements engineering and user centered conceptual design methods (namely AWARE and W2000) developed by HOC laboratory in collaboration with TEC-Lab (University of Lugano). CM suddenly showed a fervid interest in these innovative methods (w.r.t the average quality of adopted practices in the Italian web industry). From that time on, $\mathrm{CM}$ was looking for a suitable real project to experiment together our methodologies with the purpose of a future potential full adoption in other projects. At the beginning of 2004 a suitable opportunity was found, and from May to September 2004 Politecnico di Milano and CM have collaborated for the exploitation of these methods within the research project Infosfera. Within the project, these methods had to be used for demonstrating their effectiveness in supporting the development of multichannel web applications (available on a variety of fruition devices, such as PDAs, iTVs, web, and smartphones).

To validate the challenges posed by this explorative research project, Politecnico di Milano and CM decided to apply the two state-of-the-art methods to a real industrial project commissioned by the Italian Ministry of Infrastructure and Transportation: the development of the institutional web portal of the Superior Council for Public Works (SCPW from now). SCPW is the most important technical consultative body of the Italian State which is consulted in case of technical questions concerning public works of considerable amount.

The development of the institutional portal was part of a medium-size project aiming to automate the whole documental flow of the SCPW activities. Besides the

\footnotetext{
${ }^{1} \mathrm{CM}$ Sistemi is a large IT company group based in Roma working for private and public clients in a variety of market sectors including finance, assurance, public administration, electronic industry and media (www.gruppocm.it).
} 
institutional Web site, the project included the development of a document management system, the site CMS, and the migration from the previous database where the documental flow was manually performed. The project team was composed by nine CM's employees with various technical roles (PM, DBA, web designers and information architects) and two researchers (one from University of Lugano and one from Politecnico di Milano) who were in charge of the user-centered analysis and design of the institutional web site.

The project presented interesting challenges which seemed providing enough room for methodology experimentation. In fact, a variety of different stakeholders and goals were involved (from the ministry committees, to the editorial staff, to the collaborating institutions and organizations, to a diversified set of user profiles) and the project seemed therefore particularly suitable for the adoption of a goal-oriented perspective in requirements engineering. Moreover, the communication potential of the web application was rich, as rich and structured was the content to be conveyed through the interactive means. In this view, the project seemed also particularly suitable for the adoption of a structured approach to the conceptual design for the information and navigation architecture.

Keeping simultaneously one eye on the project and the other on how we used the methodologies, we tried to target three meta-objectives:

1) As to the project success, we tried to keep a stakeholder-centered perspective from requirements elicitation to prototyping, so to possibly satisfy all the stakeholders.

2) As a traceability concern, we tried to diminish the gap between requirements and design, iterating as much as possible during the process and keeping the documentation up-to-date as realistically as we could.

3) As a methodological concern, we tried to continuously monitor the capability of the existing methodologies (in terms of expressiveness and usability) of facing the challenges we encountered.

The lessons learned are interesting for different aspects. They report what the research groups (Politecnico di Milano and University of Lugano) and the industrial partner (CM Sistemi SpA) have learned from this experience in terms of practical and methodological recommendations for requirements and web design practice. Moreover, they suggest directions for improving the specific methodologies per sé and their exploitation in a real environment.

The remainder of the paper is the following. Section 2 recalls the distinctive features of the requirements and design methodologies we used in the project (respectively AWARE and W2000). Section 3 summarizes the project workflow and discusses the activities carried out. Section
4 explains and comments on examples of the requirements and design artifacts we produce along the project with the methodologies at hand. Section 5 and 6 points out key lessons learned which we consider useful for requirements research and practice. Concluding remarks and future work are presented in Section 7.

\section{Related Work}

After a first stage where pioneer approaches to web development were predominant and time-to market was the unique driving factor for the deployment of websites, nowadays practitioners are devoting more and more interest to the quality and effectiveness of final products. On one hand, these attributes are strongly related to fitness to users' and stakeholders' requirements and to the usability of the final website. On the other hand, complexity of modern websites is significantly growing up. In this light, structured and systematic approaches to requirements and design, have the potential of playing an important role for shaping effective interactive applications. However, several obstacles have been acknowledged as general barriers for technology transfer, as documented in recent industrial experience and surveys [2][5]. Although the growth of web applications, in the requirements engineering research, no significant reports on experiences have been found about the transfer to the web industry of requirements and conceptual design methods specifically tailored for communicationintensive website development.

If we look for modeling methods and tools that have gained significant acceptance in the ICT development industry, we see that UML [8] appears extensively used for modeling software modules and system architectures. However, it is still highly controversial whether UML can be used effectively to model properly stakeholders' requirements [15]. Furthermore, the lack of UML in addressing the most important issues in the design of hypermedia applications is widely documented by several extensions (a list of which can be found in [11]) that have been proposed over the last years.

To cope with the specific needs of modern Web applications, our groups have developed two methodologies - AWARE and W2000 - which root their foundations in more than a decade of experience gained working on and with HDM [9], one of the first wellrecognized design models specially tailored for hypermedia applications.

Let us briefly recall the essential features of AWARE and W2000.

AWARE (Analysis of Web Application Requirements) [6] offers simple primitives enabling to document and specify goal-oriented hypermedia requirements. It 
provides a set of conceptual tools that web analysts might find useful for describing and reasoning with website requirements. Like traditional goal-based approaches (such as $i^{*}$ or KAOS), it recognizes the central role of the stakeholders and their goals for requirements elicitation and analysis. However, whereas $i^{*}$ [12] provides constructs to model actors and their dependencies with respect to their goals and tasks, AWARE extends the analysis of $i^{*}$ to the elaboration and definition of hypermedia-specific requirements [6]. To this end, AWARE provides a requirements taxonomy to bind requirements to hypermedia conceptual design and help organize the design activity accordingly. The requirements taxonomy is used to define the so called requirement dimensions. AWARE proposes a basic set of dimensions (Content, Structure of content, Access Paths to Content, Navigation, Presentation, User Operation, System Operation, Interaction) that can be easily extended accordingly to the specific design needs.

W2000 [7][9] is a structured method for the conceptual design of the user experience. It organizes the design of a (complex) hypermedia application around four main activities:

- Information design: defining the basic conceptual information units (entities) as perceived by the user and the different access structures (collections) enabling users to reach them on the basis of their needs.

- Navigation design: defining the navigation structures basically in terms of nodes (i.e. units of interaction) and links allowing users move among them.

- Presentation design: defining the page structure in terms of lay-out aspects, graphical elements and page organization relying upon the navigation design.

- Business Process Design: defining the service architecture of a Web application in terms of processes and operations.

Following the W2000 design strategy, the conceptual design is achieved at two levels of detail: in-the-large design, where a coarse-grain solution is quickly sketched to meet initial requirements, and in-the-small design, where solutions are detailed to be used as input for the implementation activities. The path between the former and the latter levels is not straightforward but is traversed in several iterations.

\section{How the project developed}

The existing SCPW's website was an electronic brochure presenting the mission and activities of the institutions. The SCPW wanted to re-define, or better, define its online communication strategy and thus exploiting the potential of having a website to fulfil their institutional objectives. By building its online presence, SCPW needed to improve the communication effectiveness and overall usability of the website in order to widen the current target audience. An additional, but not secondary, commitment was to define suitable strategies to automate as much as possible the current editorial workflow related to the publishing and content management activities of the website. CM was in charge of the whole project (being the winner of a public competitive examination) and, exploiting the existing cooperation with Politecnico di Milano in the context of the Infosfera project, decided to adopt and thus to verify on the field the potential of the learned methodologies.

\subsection{Overview of the SCPW}

SCPW is the most important technical deliberative body of the Italian Government, which is consulted for a variety of technical questions concerning building construction. The SCPW structure includes a President, who heads the overall structure, six thematic sections, a general assembly and an independent body called "Servizio Tecnico Centrale" ("Central Technical Service", hereafter STC). Each section is specialized for a specific field of public works (e.g. Section 1: building trade; Section 2: hydraulic trade; etc.) while the general assembly is consulted in case of works of extraordinary relevance. Sections and assembly have a consultative purpose: the result of their decisions does not take the form of a mandatory rule. Unlike sections and assembly, the STC activity is operative, in that it is called to define technical constraints and procedures, to deliver certifications, to carry out inspections and procedural vigilance in the arena of building construction. STC is independent from the sections and the assembly but it is directly subordinated to the President. In order to answer an important issue concerning technical questions made by other public bodies or citizens (e.g. a civil engineer association wondering whether it is allowed to use a new alloy for the roof of public buildings), the corresponding SCPW section organizes a consultative meeting (depending on the question, the meeting may involve more than one sections) whose result is a public "Opinion" (in Italian "Parere") about the question. Besides these main activities, the SCPW is in charge of other minor tasks like contributing to the definition of normative laws, answering to professionals, to citizens' or companies' questions about their everyday activities, pointing to already-answered questions, resources, and existing laws on the matter at issue.

\subsection{Project workflow}


The starting point of any public work is often the contractual specification which is provided to the competing companies for formulating the offers. The initial specification for this project was written in natural language. Although rich in functional details, it was quite vague regarding the motivating institutional goals of the project, which are typically the main source of information for defining a proper communication strategy. In this context, we started our analysis process following the approach depicted in Fig. 1. The first two phases (Project set-up and Preliminary Analysis) initialize the overall project activities, while the following ones form a loop that is executed a number of times and represent the core of the iterative analysis process.

Project set-up is a phase in which a common ground about the methodologies to-be adopted is set. To this end, we provided a intensive crash-course on both our methodologies. Our strategy consisted in teaching the methodology philosophy and the main concepts in a short time (four full days) discarding details and possible variants. After this first course, the designers were able to design a simple application exploiting the learned concepts. After this preliminary training, we worked side-by-side with learning practitioners in their project. During the project experience most of the remaining details and variants were introduced and discussed when needed. In the meantime, the full methodological material and cases studies had been made available to the practitioners [6].

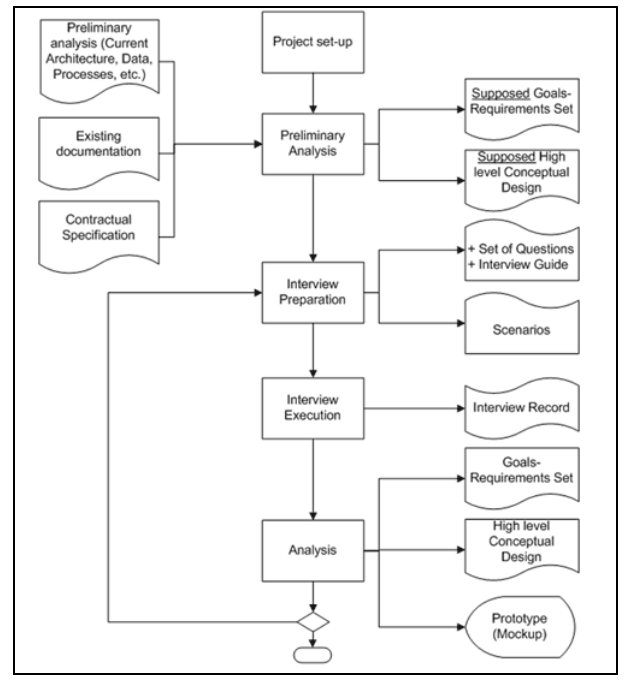

Figure 1. Project Workflow

During the Preliminary Analysis the existing documentation, the contractual specification, the current editorial workflow, and the existing website are analyzed. On the basis of this analysis, a set of supposed requirements (using AWARE) and corresponding design specification (using W2000) have been defined. We call these preliminary models "supposed" because their main purpose is to reshape the available information in a form more suitable for an engineering analysis but they do not aim at representing the actual requirements and design. In this phase the requirements set and the design are generally fragmented and characterized by several ambiguities to be investigated.

At this stage, a first high level conceptual design is also produced to reify supposed requirements in a form suitable for discussing with other people. Discussing about design aims at determining connections between pieces of design and underlying requirements, and at discerning among possible solutions or variants of preliminary ones. This kind of design should be opened up enough to be modified on the fly during a discussion and lightweight enough so that little effort is needed to be reproduced.

As showed in Fig.1, after the Preliminary Analysis an iterative elicitation activity starts up. Three interviews were the core of this activity and iteratively triggered the following tasks: an interview preparation phase, where the current results are used to prepare the next elicitation activity; an interview execution phase, where new information is gathered; an analysis phase, where the acquired information are matched against the current requirements and design in order to develop a refined version of both of them.

Due to organizational reasons, the two first interviews involved two section's heads, the vice-president, a secretary (usually in charge of the editorial activities) and the STC's head; the President, the most influential stakeholder, participated in the third interview only.

Thanks to this iterative revision, requirements and design tended to be more comprehensive and better related each other. As far as the iteration goes on, the design is also tuned and detailed moving from the in-thelarge level to the in-the-small one. Passing from a level to the other some design decisions could be revisited and new requirements emerge. Moreover, comparing the preliminary analysis and the final analysis results, it can be noticed that a new type of output is added: a prototype. The purpose of the prototype in this process is to put the design solutions in a form suited for discussing with the client. Our strategy in this phase is to show the prototype in the form of visual page mock-ups as a base for the discussion, thus hiding the design models behind.

As to the involvement of potential users in the process, we contacted some professional associations in the preliminary analysis phase, succeeding in interviewing some of their members. However, due to organizational reasons, it was not possible to meet them again when a prototype was available. In our project 
experience, this kind of situation is very common (almost the standard) in Web application development process, since potential users do not belong to the client company and thus are hard to reach.

\section{Analysis Artifacts}

\subsection{Stakeholders}

A number of stakeholders had to be taken into account to devise an effective communication strategy for the institution and deliver a usable application. These stakeholders cover some recurrent roles common to projects concerning institutional communication even of greater complexity. Figure 1 shows a classification of the website's stakeholders for SCPW.

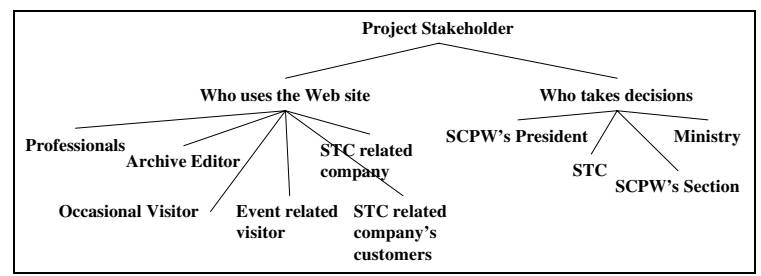

Figure 2. Taxonomy of Website stakeholders.

Among the site's visitors: "Professionals" are technical people (engineers, architects, works managers, public body's agents, etc.) who work in the building construction field. "STC related companies" are those companies whose work strongly depends on normative documents produced by the STC (e.g. every year a geologic laboratory has to renew its authorization to release results on the status of an area: the STC defines and publishes on the website the procedure and the constraints for authorization renewal). "STC related company's customers" are privates or other companies who are customers of companies whose activity is subordinated to some public authorization. "Occasional visitors" are those who have heard about the SCPW in some way and may want to understand whether or not it may be useful for their activity. "Event-related visitors" are those who are waiting for a specific activity to be accomplished (e.g. an assembly and the relative "opinon" to be published). "Archive editors" are SCPW's employees who are in charge of adding new documents to the website's archive.

As to the institutional main stakeholders, the decision makers are (in decreasing order of decision power): the "Ministry of Infrastructure and Transportation", the "SCPW's President", the "STC responsible", and the various "SCPW's sections". The ministry can be considered as an "abstract" stakeholder in the sense that his decision power and vision are embodied in the SCPW
President, who was the only interface between the analysts and the Ministry.

\subsection{Goals and Website Requirements}

We now discuss some excerpts from the user requirements analysis, which presents interesting material to learn from the analysis process. Except for the Archive editors, all user categories have two very generic and abstract goals for the website: understanding what the SCPW (and the STC) can offer and finding out the needed information. However, these goals assume a different meaning as the user category varies, bringing to different sub-goals and requirements. In Figure 3 and 4 the goals and requirements of two user categories are depicted.

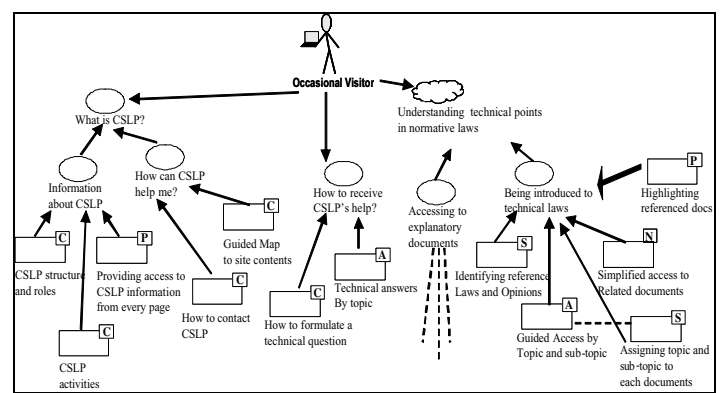

Figure 3. A piece of AWARE analysis for Occasional User: from goals to requirements. For a detailed legenda see [6].

Only occasional visitors need some general information about SCPW, because it has been assumed (and verified during the interview with SCPW) that professional users already know very well how SCPW may be useful for their daily work. To meet the goal "What is SCPW" of the occasional visitor, two content and a presentation requirements are devised (in Figure 3, CSLP [Italian] stands for SCPW).

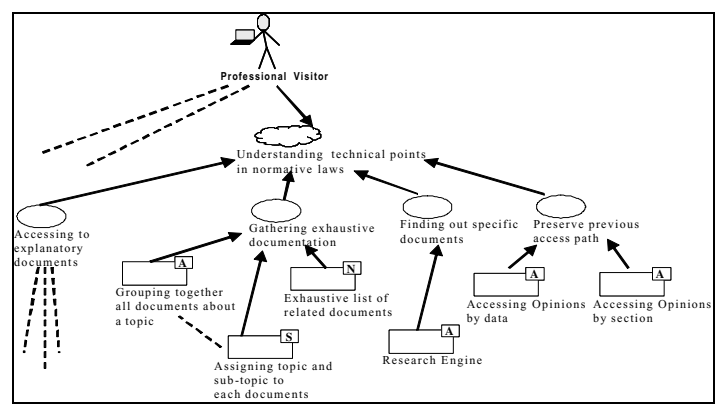

Figure 4. A piece of AWARE analysis for Professional user: from goals to requirements.

They state that information about SCPW and its activities should be provided to first-time users enabling 
them to take out a clear picture of how SCPW may help them and in which way (content requirements).

Moreover, since these users are not familiar with the website and they could land from the Internet to any internal site page, a link towards this general SCPW content should be always available and evident (presentation requirement).

As to the shared soft-goal "Understanding technical points in normative laws" the refinement analysis makes evident the different needs behind this common goal. Both users aim at "Accessing explanatory documents" (requiring several kinds of documents, as "Opinions", "Laws", "Specification templates", "Guidelines", "Technical answers", etc.). However, the main difference is that occasional visitors do not have a clear idea about the content of the Website and needs to be guided, introduced and helped out to discover the documents which may be useful to them. On the contrary, professional users know very well what is offered by SCPW, are accustomed to the Website (since they are usually highly recurrent users) and need sophisticated searching mechanisms for retrieving specific documentation. Among other requirements, this goal bears the need for a classification of documents by topic. This need is refined in two kinds of requirements, a structural one that entails topics and sub-topic being included in the documents structure, and an access requirement that asks for some access paths that exploit the topics classification.

Summarizing the key elements of the analysis artifacts, the whole requirements set consists of 28 content, 16 access, 5 structure, 5 navigation and 4 presentation requirements.

\subsection{Conceptual Design}

The conceptual model focuses first and foremost on the user experience, i.e. on how designers want the application to behave from the perspective of the end users, thus temporarily neglecting the description of details related to the specific technologies and implementation. In this sense, W2000 provided a seamless path from the requirements set to the usercentered conceptual design.

Looking at the classified requirements summary, a clear picture of the application design can be figured out. Observing that the largest sets of requirements are content and access requirements, we can derive that most of the design effort should be put in designing information objects (entity types in W2000 terminology) and ways of accessing and locating the content (access structures in W2000 terminology). The remaining requirements (concerning navigation/interaction, structure and presentation) are not particularly constraining and leave the designer a higher level of freedom in shaping the user experience. The following examples are taken from the overall design documentation in W2000. Their main purpose is to show W2000 in action and its relation with the requirements set. One of the most important entity types which has been devised to the new version of the website is the "Technical law". In Figure 5, the in-the-small description of this information object is reported as extracted (and translated) from the design document. In the first iteration of the analysis process only multiplicity, semantics, source and comment have been described, with several references to the recorded interviews, to goals and requirements.

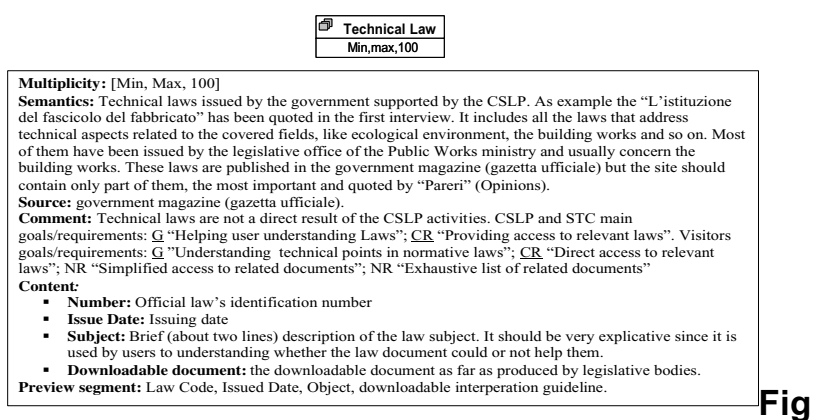

ure 5. In-the-small description of entity type: "Technical law".

As to the relation with requirements, the "semantics" and "comment" field provide explicit pointers to goals and requirements documented in the analysis. Here, we do not use a formal traceability technique to take into account requirements in the design artifacts, but we rather fill in informally these fields using a syntax like the one showed in Fig. 5: "G" for goal, "CR" for content requirement, "NR" for navigation requirement, and so on.

As to the navigation design, one kind of navigation connection among information object types (semantic association in W2000 terminology) is worth noticing. The semantic association "Useful References" is a path enabling to navigate from a document to a related one with two possible semantics (see Fig. 6). The association has been designed for supporting navigational requirements that required some navigable connections between documents with the purpose of helping the user to better understand the documents available (e.g. G "Understanding technical points in normative laws"; NR "Simplified access to related documents"; NR "Exhaustive list of related documents"). From the content management point of view, we decided that when the archive editor inserts a new document in the website, $\mathrm{s} / \mathrm{he}$ should also specify some reference documents. In defining this association, we acknowledged an advantage 
deriving from the use of W2000. Since in W2000 all associations are considered bidirectional by default (except if explicitly decided differently), a question was immediately made by the designers when designing this navigation path: which is the semantic of the opposite direction?

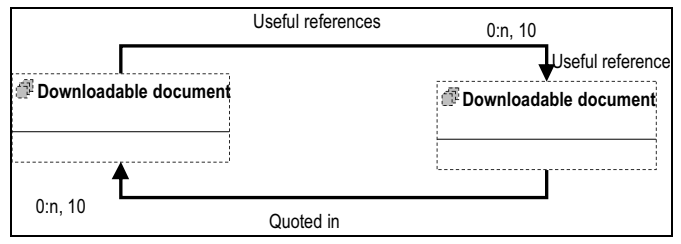

Figure 6. Example of semantic navigation design.

As shown in Fig. 6, we assigned to the opposite direction the meaning of "Quote". Thus, if a document $X$ has a document $\mathrm{Y}$ as reference, then $\mathrm{Y}$ has $\mathrm{X}$ as quote. Thus, when a user finds out an interesting document, $\mathrm{s} /$ he can find not only a list of referenced documents, but also a set of documents which quote it as reference, expanding the correlation possibilities.

Two immediate advantages derive from this decision: from the content management point of view, no effort is required to the Archive editor, since quoting documents are automatically derived. Moreover, looking to the size of the list of quotes, a user can also figure out the importance of the current document, thus being supported in achieving the goal "Being introduced to technical laws" (see Figure 3).

Once content has been designed by means of entity types, the next step in the W2000 method is to define how user can reach such content, that is, the access structures. Access structures are modeled by collections of entities or other collections. A collection containing another collection defines an access path, while a collection containing only entities is called base collection and its members are instances of the entity type selected on the basis of some population criteria.

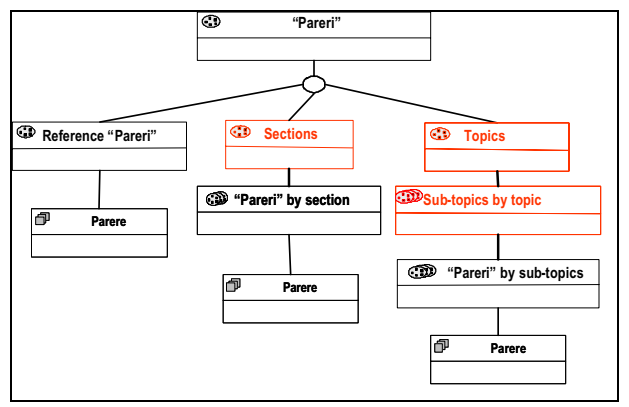

Figure 7. Access structures to "Opinions" (it. Pareri).
In Figure 7 the collections designed to access the instances of the entity type "Opinion" (in Italian "Parere") are depicted. Looking at these collections we can notice as the access requirements shown in the previous paragraph have been resolved at this design stage. The access requirements stating the need of organizing the "Opinion" by topic and by section gave raise to specific nested collections which are accessible from a starting point of all opinions. The actual topics have been defined together with the stakeholders The last step in the design process is the definition of pages structure and content, shaped on the basis of the information and navigational units defined in the earlier design activities. For space reasons, we cannot here report sketches of the page prototypes and discuss in detail the design decisions taken for page design. For a more in-depth explanation, please refer to [14]. The final published website can be browsed at: $\mathrm{http} / / / \mathrm{www}$. infrastrutturetrasporti.it/consuplp/.

\section{Lesson Learned on behalf of the research group}

In this section we discuss some considerations, from the academic-to-industry point of view, in form of lessons learned which, we hope, could help in similar projects. To this end, we have tried to abstract from the specific characteristics of the project. Lessons learned are classified as general (G1 and G2), methodological (M1, $\mathrm{M} 2$, and M3) and technique-specific (T1 and T2).

Lesson G1: Web industry needs for proper "mediators" to cope with emerging technologies.

As mentioned in [5], industry often does not employ systematic approaches, like those usually promoted by the academy, and does not retain an appropriate knowledge. In most of cases, requirements and conceptual design are only sketched in natural language without following any method. However, the increasing request for quality and effectiveness is driving the web industry towards the research of such approaches. In this light and due to the inherent complexity of structured methodologies, a proper mediation of experts is required, in order to avoid novice practitioners feeling lost and isolated (overcoming the "negative perception" as defined in [5]). A possible mediation approach - which turned out to be effective in our experience - is to provide the basics of the methodologies in short and intensive training sessions, and then carrying out together (trainers and trainees) case studies (possibly real-world ones) in their known domain.

Lesson G2: Modularity and flexibility of the methods can help overcome organizational obstacles for adoption. 
In general, internal organization and current practice of target companies allow for new technologies to be only partially used, reducing their potential impact. On the other hand, it is difficult to ask for a drastic change in the proven practice of a company without seriously compromising the chances of convincing them to try the new approach. The introduction of new methods should be carefully matched with the current organizational habits in dealing with projects. Besides the any specific "mediation" strategy adopted (as recounted in lesson G1), in our experience (and in particular in this project) we found two key requirements for the introduction of a web methodology in the industry: modularity and customization. Modularity means that analysts, designers or developers should be put in the conditions to take the part they wish (of the method), not being forced to "all or nothing", and still being able to use it with some observable advantages [5]. As to customization, a methodological suite should be enough flexible to adapt to the specific needs of the organization - such as the presence of existing development tools, documentation habits, decision structures, work organization and task assignments - and be used effectively.

\section{Lesson M1: Interviews to potential website users can} rarely be performed in practice.

The satisfaction of the user experience plays a crucial role for the final success of a website. Unfortunately, organizing interviews or other forms of requirements elicitation enabling a direct contact with the final users is very expensive, especially when done iteratively. To supply to this deficiency, user requirements are often simulated or put forth by some stakeholders belonging to the client company. This should not seem so out of scope, since often stakeholders know, from their everyday experience, the typical behavior and needs of their current clients and can therefore provide insightful knowledge to the analysts. However, it is important to carefully examine the gathered material in order to avoid dangerous biases (current stakeholders' clients do not cover the entire spectrum of potential website users!). In our case, the interviewed stakeholders were specialized in different technical areas of the SCPW. This sample was quite good for simulating the needs of "professional visitors" but rather unreliable for the "occasional visitors". To bridge this elicitation gap, project analysts and designers relied on their past experience in similar web project to "prompt" candidate user requirements and validate them with the stakeholders.

Lesson M2: Web communication requirements may be controversial across different branches of an institution.

Communication requirements are a very delicate matter, which have a strong impact on how each part of the institution perceives itself and wish to communicate its image to the public, and on how the image of the institution in its whole is shaped. In our project, we elicited by STC's head that STC had to appear as the main technical body within SCPW. So, our first specification contained the presentation requirement "Providing users with direct access to STC's services". As a consequence, our first design solution was to have the website home page in a way that STC services gained preferential access paths, apart from the rest of SCPW's activities. This requirement was about to be broadly accepted by all other interviewees until the SCPW's President (in the third interview) strongly disapproved this solution in the first prototype, clearly stating that the goal was to "Communicate a unitary and coherent image for all the SCPW activities", without favoring any internal department more than others. From our errors, we learned that it is recommendable to interview firstly the main stakeholders who have the highest decision power on the communication strategy, and then the ones who may be not consistent or aligned with this vision. Moreover, the elicitation process should involve, in a balanced way, the overall organization, being aware as early as possible about the different points of view, and taking into account the relative power of various stakeholders. In our project, the highest importance was assigned to the President, who imposed a change in the communication requirements, which in turn were operationalized in a change in the home page design.

Lesson M3: To gather richer feedback during requirements validation, a "tangible" artifact is preferred.

Due to the abstract nature of goals and requirements, a validation discussion solely focused on requirements models turned out to be ineffective and ultimately misleading. The main reason for this relies in the abstraction level in which requirements are expressed. Discussing about needs is sometime too abstract to obtain a concrete feedback. Since the first interview, we gathered the need of making available through the site a type of document called "technical answers" (produced by the STC). Also in the second interview (based upon the first requirements model) this kind of information seemed as equally important as the other kinds of documents ("opinions", "technical laws", "guidelines", etc.). From these requirements, our first design solution was to have similar access structures for all kinds of documents, including the "technical answers". Only after the first prototype (provisional web pages on which this solution was encoded), our idea was rejected, opting in favor of a prominence for "Technical Answer". Prototypes can greatly help in communicating provisional solutions to stakeholders and to gather reliable feedback for validating the requirements. The input gained can be then fed into structured requirements 
formalisms for appropriate analysis and refinement. The iterative interplay between goal-oriented elicitation, highlevel conceptual design and prototyping is crucial for refining the requirements set. Requirements, in particular, as intended in this paper, provide an abstraction from the specific solution (which may risk to make stakeholders to focus on irrelevant details) so that strategic decisions for the project can be determined; conceptual design can be used to materialize abstract needs into possible solutions, which may be then shaped into a tangible prototype [13].

Lesson T1: AWARE is still too rigid to stimulate and creatively support the elicitation and analysis process.

In this project AWARE has been used to specify supposed goals and requirements during a preliminary analysis phase. Here, the constructs offered by the model provide the conceptual elements to interpret the documentary material available, to extract the relevant goals and requirements to be further elaborated and discussed, and to give a logical and reasoned structure (still provisional) to the requirements picture. During the iterative elicitation process, analysts gradually changed the AWARE artifacts to meet the new requirements and relations emerged, until a coherent and reliable version was produced to be fed to designers. Although powerful in modeling, AWARE should provide more process guidelines and tips on "when to do what". For example, a guided process on how and when to "prompt" requirements for web applications would have been a major support for the work of the analysts. In this sense, AWARE turned out to be still too rigid to provoke scenarios, and prompt examples with stakeholders to discuss and create new requirements, or to point out lacking pieces of the analysis picture.

Lesson T2: W2000 is good for specifying fully developed design solutions but of little use for generating ideas.

W2000 is an effective model for describing in details and in a coherent fashion the needed web design solution to meet a given set of requirements. Practitioners acknowledged that the structured and complex apparatus of the W2000 methodology is a valuable guidance for designers not to forget about important design issues, which would be otherwise neglected, thus compromising the quality of the application. We learned once again from this experience that a web design model should be used for brainstorming, i.e. for generating and discussing ideas among developers, with stakeholders, and with potential users. It is of little use to have a design model capable of representing only fully developed solutions. Even an incomplete design is an important artifact to be produced, since it can be quickly produced and validated in order to trigger ideas, discard possibilities and making new requirements surface. Moreover, it must require little time to write down design ideas (an activity which now takes too long with W2000): developers do not like to spend too many resources in preliminary activities.

\section{Lessons learned on behalf of the industrial partner}

In this section we summarize some considerations pointed out by the CM team during the project and the training days.

Lesson 1: The availability of systematic web design methods is vital to structure team work activities.

Team organization and activity scheduling for complex projects are negative affected by the lack of structured design activities. Such a problem is emphasized in the scenario of complex interactive applications, where the separation between the different aspects of the systems (e.g., contents from navigation, from structure, from presentation and from technological architecture) is a quality/cost key factor. The approach proposed by W2000 effectively addresses this need, and could be systematically adopted to increase the quality of the development/maintenance projects. Moreover, W2000 structured approach also enabled the project team to systematically cope with usability issues early in development (even before a prototype is available) because the method offers a clear description of the design artifacts and suggests proven (usable) design patterns to adopt [16]. Note that this lesson may be seen in contrast with T2. This is due to the fact that they represent two different perspectives on the use of W2000 (the one of the researchers and the one of the developers). On one hand, according to the researchers, W2000 is still too close to detailed design specifications and it is rigid to provoke and support creative brainstorming. On the other hand, according to the developers - who did not have previous experience with any other user-centered design method - , W2000 is "better than nothing", thus being a great step ahead in this direction.

Lesson 2: In projects where time-to-market is vital, semi-formal approaches are preferred to formal ones.

A trade-off between quality and time-to-market is crucial in web projects. Although clients increasingly ask for quality applications (where the achievement of the institutional goals and the quality of the user experience are major concerns), they are still reluctant to spend time in trying to understand complex and formal requirements documents. Besides the client's background (which is often non-technical), the main reason for that is the timeto-market, which is an inevitable (fortunately not the only one) driving factor for website development. Semiformal approaches (as W2000 and AWARE) which 
combine natural language descriptions, sketches, prototype design and commented requirements schemas seem to be quite accepted by non-technical stakeholders because of their efficiency and understandability.

Lesson 3: Adequate support tools are needed to fully exploit the potential of structured requirements and design methods.

Although very powerful and effective for the modeling activity, AWARE and W2000 have still not adequate tool support. Support tools might partly mitigate the issue explained in the previous lesson, providing analysts with an efficient and consistent way to deal with structured requirements and design approaches. Tools should also enhance the documentation quality and thus facilitate stakeholder's understanding. Effective modeling approaches corroborated by appropriate tool support will enable industry to implement interactive solutions in a competitive way.

\section{Conclusions and Future Work}

We have synthesized and discussed the experience gained during the development of an institutional web application (the website of the Superior Council of Public Works of the Italian Government), in which a research group (HOC lab from Politecnico di Milano and TECLab from the University of Lugano) together with an industrial IT partner (CM Sistemi) have employed structured requirements and design techniques to face the challenges of the project. In particular, a goal-oriented requirements analysis method (AWARE) was coupled with a structured approach to user-centered conceptual design (W2000). Besides trying to transfer this knowledge to the industrial partner collaborating to the project, we had the opportunity to verify on the fields the advantages and limits of the approaches and to gather methodological and practical recommendations for future projects in similar domains. Future research will consolidate the methodologies in the effort of making them more lightweight (thus easier to teach), more suitable for brainstorming (rather than for describing fully developed solutions) and more usable by web practitioners. Moreover, proper tool support for AWARE and W2000 will be designed and enhanced for facilitating practitioners in the analysis and documentation process.

\section{Acknowledgments}

The authors are grateful to all people from $\mathrm{CM}$ Sistemi who participated in the training sessions in Roma for their insightful feedback on the methodologies
AWARE and W2000. Thanks also to the people at CM who coordinated and finalized the project.

\section{References}

[1] M. Jackson, "The World and the Machine", a Keynote Address at ICSE-17; in Proc. of ICSE-17, ACM Press, 1995.

[2] C. Barry, M. Lang, "A Survey of Multimedia and Web Development Techniques and Methodology Usage", IEEE Multimedia, April-June, 2001.

[3] C. Britton et al., "A Survey of Current Practice in the Development of Multimedia Systems", Information and Software Technology, vol. 39, no. 10, 1997, pp. 695-705.

[4] F. Garzotto, V. Perrone, "On the Acceptability of Conceptual Design Models for Web Applications", in Proceedings of ER'03 Workshops, International Workshop on Conceptual Modeling Quality (IWCMQ'03), October 2003 , Skokie, Illinois, USA.

[5] H. Kaindl et al., "Requirements Engineering and Technology Transfer: Obstacles, Incentives and Improvement Agenda". Requirements Eng. 7(3): 113-123 (2002).

[6] D. Bolchini, P. Paolini: "Goal-Driven Requirements Analysis for Hypermedia-intensive Web Applications", Requirements Engineering Journal, Special Issue RE03, Springer 2003.

[7] L. Baresi, F. Garzotto, P. Paolini, and V. Perrone, "Hypermedia and Operation Design". Deliverable D7 of the European IST project UWA (Ubiquitous Web Applications), www.uwa-project.org.

[8] G. Booch, I. Jacobson, and J. Rumbaugh, "The Unified Modeling Language User Guide". The Addison-Wesley Object Technology Series, 1998.

[9] F. Garzotto, P. Paolini, D. Schwabe, "HDM - A ModelBased Approach to Hypertext Application Design". ACM TOIS, Vol. 11, No. 1, January 1993.

[10] P. Fowler, M. Patrick, A. Carleton B. Merrin, "Transition package: an experiment in expediting the introduction of requirements management", in proceedings of ICRE 98.

[11] V. Perrone, "Conceptual Modeling of Multi/Cross channel Web Applications", $\mathrm{PhD}$ dissertation thesis, Politecnico di Milano, 2004.

[12] E. Yu, "Modeling Organizations for Information Systems Requirements Engineering", in Proc. 1st Int. Symposium on Requirements Engineering, RE'93, San Jose, USA, 1993.

[13] R. Paiano, A. Pandurino, "WAPS: Web Application Prototyping System", in Proc. of 4th International Conference, ICWE 2004, Munich, Germany, July 26-30, 2004.

[14] V. Perrone, B. Bolchini, Progettazione e realizzazione del sito Internet e della Intranet del Consiglio Superiore dei Lavori Pubblici, TEC-Lab Technical Report, TR05.1, TEC-Lab, University of Lugano, 2005.

[15] C. Stutz, J. Siedersleben, D. Kretschmer, W. Krug, "Analysis beyond UML", in Proc. IEEE Joint International Conference on Requirements Engineering, RE'02, Essen, Germany, 2002.

[16] Paolini, P., Garzotto, F., Bolchini, D., Valenti, S., 'Modelling by Pattern' of Web Applications, in Proc. ER'99 World Wide Web and Conceptual Modeling, Paris, France, November, 1999. 